\title{
Qualitative and quantitative analysis of catechin and quercetin in flavonoids extracted from Rosa roxburghii Tratt
}

\author{
Ming-Hua Hao ${ }^{1}$, Fan Zhang ${ }^{1}$, Xing-Xia Liu ${ }^{2}$, Fan Zhang ${ }^{1}$, Li-Juan Wang ${ }^{1}$, Sai- \\ Juan $\mathrm{Xu}^{1}$, Jin-Hua Zhang ${ }^{1}$, Hong-Long $\mathrm{Ji}^{3}$, Ping $\mathrm{Xu}^{1 *}$ \\ ${ }^{1}$ Department of Pharmacy, ${ }^{2}$ The Third Affiliated Hospital, ${ }^{3}$ Institute of Lung and Molecular Therapy, Xinxiang Medical University, \\ Xinxiang, Henan, 453003, China
}

*For correspondence: Email: 13273730271@163.com; Tel: +86-18240691447; Fax: +86 3733029879

\begin{abstract}
Purpose: To perform a qualitative and quantitative analysis of catechin and quercetin in flavonoids extracted from Rosa roxburghii Tratt.

Methods: Total flavonoids were determined using ultraviolet spectrophotometry (UV) at $500 \mathrm{~nm}$. The optimal gradient program started with $15 \%$ methanol and was kept within a period of $0-20$ min, while $25 \%$ methanol was kept within $20-33$ min. Subsequently, the concentration of methanol was reduced to $15 \%$ and was held for 10 min until the next injection. Mass spectrometry spray voltage was 4,000 V, ionization temperature $350{ }^{\circ} \mathrm{C}$, atomizer pressure $35 \mathrm{psi}$, nitrogen flow rate $8 \mathrm{~L} / \mathrm{min}$, and mass scan range $200-800 \mathrm{~m} / \mathrm{z}$. The detection wavelength used for catechin and quercetin was 270 and $368 \mathrm{~nm}$, respectively.

Results: Based on the UV results, Rosa roxburghii Tratt content was $73.85 \%$, which is in agreement with the national standard. Liquid chromatography-mass spectrometry (LC-MS) results indicate that Rosa roxburghii Tratt flavonoids contained quercetin, $34.26 \%$, with relative standard deviation (RSD) of $2.88 \%$ and catechin content of $2.97 \%$ with RSD of $1.49 \%$.

Conclusion: The proposed measurement method for determining the content of flavonoids in Rosa roxburghii Tratt has the advantage of simplicity, feasibility, good repeatability, and rapid and accurate analysis.
\end{abstract}

Keywords: Rosa roxburghii Tratt, Flavonoids, Catechin, Quercetin

\begin{abstract}
This is an Open Access article that uses a funding model which does not charge readers or their institutions for access and distributed under the terms of the Creative Commons Attribution License (http://creativecommons.org/licenses/by/4.0) and the Budapest Open Access Initiative (http://www.budapestopenaccessinitiative.org/read), which permit unrestricted use, distribution, and reproduction in any medium, provided the original work is properly credited.

Tropical Journal of Pharmaceutical Research is indexed by Science Citation Index (SciSearch), Scopus, International Pharmaceutical Abstract, Chemical Abstracts, Embase, Index Copernicus, EBSCO, African Index Medicus, JournalSeek, Journal Citation Reports/Science Edition, Directory of Open Access Journals (DOAJ), African Journal Online, Bioline International, Open-J-Gate and Pharmacy Abstracts
\end{abstract}

\section{INTRODUCTION}

Rosa roxburghii Tratt is a Rosaceae deciduous shrub of the genus Rosa, and is also known as the silk flower. The nutritional values of Rosa roxburghii Tratt are extremely high. Indeed, this fruit is rich in natural antioxidants such as flavonoid compounds, vitamins, and superoxide dismutase [1-4]. In addition, Rosa roxburghii Tratt fruit is reported that it has multiple functions such as improving the immune function, antiatherosclerosis properties, lowering blood 
pressure, eliminating free radicals, and reducing lipid over-oxidation injury [5-9]. The efficacy of Rosa roxburghii Tratt extracts have been evaluated in pancreatic cancer [10], human gastric cancer cells, and human liver cancer cells $[11,12]$. It was reported that Rosa roxburghii Tratt has also beneficial effects on liver cells [13].

Rosa roxburghii Tratt contains many flavonoid compounds [5]. Of these, catechin has been reported to exert several beneficial properties, such as scavenging free radicals, anti-mutation properties, radioprotection, inhibition of anaerobic bacteria proliferation, and reduction of high cholesterol [14,15]. Several assays to measure catechin have been reported $[16,17]$. In addition to catechin, quercetin is another flavonoid of Rosa roxburghii Tratt (FRT) and has been reported to lower blood pressure, improve blood capillary resistance, reduce capillary fragility, decrease hematic fat, and increase coronary blood flow [18].

A high performance liquid chromatography (HPLC) method to evaluate catechin and quercetin qualitatively, but no quantitatively, has been reported $[19,20]$. Therefore, in the present study, we tested the use of a reverse HPLC method to measure both FRTs quantitatively.

\section{EXPERIMENTAL}

\section{Reagents and equipment}

The following reagents were used: Rosa roxburghii Tratt (Henan Kaifeng Medical Building, China) and purified catechin and quercetin (Institute for Food and Drug control, China) with at least $98 \%$ purity. Furthermore, the following equipment was used: Agilent 1260 HPLC; quaternary pump; diode array detector; autosampler; Agilent Chem Station chromatography workstation; Senco R205B rotary evaporator (Shanghai Shen Sheng Technology Co. Ltd, China); Hangping-FA 1104 electronic balance (Shanghai Balance Instrument Factory, China); 202-2-BS electric thermostatic drier (Shanghai Yuejin Medical Instrument Factory, China).

\section{Extraction and separation of FRTs}

Rosa roxburghii Tratt was milled into powder and FRTs were extracted using $70 \%$ ethanol at approximately $90{ }^{\circ} \mathrm{C}$, followed by condensing and refluxing for $4 \mathrm{~h}$. The filter residue was discarded, and the filtrate was evaporated using a rotary evaporator. Subsequently, ethanol was removed using the rotary evaporator, and the samples were dried and weighed. A glass packed column served for leak detection.
Subsequently, the sample was applied to an HPD 600 macroporous resin-packed column, followed by $4 \% \mathrm{HCl}$ and $4 \% \mathrm{NaOH}$ activation pillars. After adsorption for $1 \mathrm{~h}$, distilled water was used to remove impurities. Subsequently, desorption was performed by washing with $40 \%$ ethanol eluent, followed by ethanol removing, and sample drying and weighing.

\section{Determination of total FRTs by ultraviolet (UV) spectrophotometry}

Rutin $(2.12 \mathrm{mg}$ ) was dissolved in methanol and placed in a 10-mL volumetric flask. Subsequently, the dissolved rutin volumes (concentration) of $0 \mathrm{~mL}(0 \%), 0.4$ (16.67\%), 0.8 (33.33\%), 1.2 (50\%), 1.6 (66.67\%), 2.0 (83.33 $\%)$, and $2.4(100 \%)$ were transferred to a new 10-mL volumetric flask; the absorbance $(A)$ was subsequently determined. A standard curve was constructed using $(A)$ as the ordinate and rutin concentration (C) as the abscissa. An amount of $19.2 \mathrm{mg}$ of powdered FRT was dissolved in 25 $\mathrm{mL}$ of methanol. Subsequently, $0.4 \mathrm{~mL}$ of dissolved FRT sample solution was placed into a 10-mL volumetric flask; (A) was determined at $500 \mathrm{~nm}$.

\section{Determination of quercetin and catechin by HPLC}

\section{Chromatographic conditions}

A Biopearl-HC C18 column $(4.6 \mathrm{~mm} \times 200 \mathrm{~mm} \times$ $5 \mu \mathrm{m})$ was used. The mobile phase consisted of methanol (solvent A) and pure water (solvent B), and the flow rate was $1.0 \mathrm{~mL} / \mathrm{min}$. The optimal gradient program started with $15 \%$ methanol and was kept in the 0-20 min period, while $25 \%$ methanol was kept in the 20-33 min period. Subsequently, the concentration of methanol was reduced to $15 \%$ and was held for $10 \mathrm{~min}$ until the next injection. The column oven temperature was maintained at $30{ }^{\circ} \mathrm{C}$, and the sample injection volume was $20 \mu \mathrm{L}$.

\section{Catechin and quercetin standard reference solution preparation}

Twenty-five milligrams of dried catechin and quercetin standard materials were weighed precisely, placed in a $25-\mathrm{mL}$ volumetric flask, and dissolved in methanol at a concentration of 1 $\mathrm{mg} / \mathrm{mL}$. The following amounts of standard solutions were placed in a $10-\mathrm{mL}$ volumetric flask: $0.1,0.2,0.4,0.8,1.6$, and $3.2 \mathrm{~mL}$; the volume was adjusted with methanol, and the solutions were mixed by shaking. 
A total of $20 \mu \mathrm{L}$ of the different reference solutions were injected into the drawing precision HPLC peak area as described above in order to measure catechin and quercetin. The standard curve was drawn with the peak area $(\mathrm{y})$ as the ordinate, and the concentration of the reference substance solution $(x, \mu \mathrm{g} / \mathrm{mL})$ as the horizontal coordinate. The regression equations of catechin and quercetin were $y=8692.6 x-55.04(r=$ $0.9997)$ and $y=31738 x-24.004(r=0.9996)$, respectively. At concentrations of $10-320 \mu \mathrm{g} / \mathrm{mL}$ $(r=0.9997)$ for catechin and $10-200 \mu \mathrm{g} / \mathrm{mL}(r=$ 0.9996) for quercetin, the two flavonoids showed good linear relationship with the peak area within the scope.

\section{Determination of quercetin and catechin in the sample solution}

An amount of $6.4 \mathrm{mg}$ of powdered FRT was weighed accurately, and subsequently added to $32 \mathrm{~mL}$ of methanol and $8 \mathrm{~mL}$ of hydrochloric acid to get a final concentration of FRT of $0.2 \mathrm{~mol} / \mathrm{L}$. The solution was hydrolyzed in $90{ }^{\circ} \mathrm{C}$ for $1 \mathrm{~h}$. Subsequently, the solution was allowed to cool down immediately in ice water for $5 \mathrm{~min}$, transferred to room temperature, and the volume was adjusted up to $25 \mathrm{~mL}$ with pure methanol. The solution was filtered using a $0.45-\mu \mathrm{m}$ organic membrane. An amount of $20 \mu \mathrm{L}$ was injected to determine the content of the ingredients.

\section{Precision test}

Catechin or quercetin solutions were prepared at $40 \mu \mathrm{g} / \mathrm{mL}$, and the peak area was determined using $20-\mu \mathrm{L}$ volumes with five repetitions. The relative standard deviation (RSD) of catechin and quercetin was 1.04 and $0.95 \%$, respectively, indicating a good measurement precision.

\section{Repeatability test}

The extracted FRTs were divided into five separately prepared solutions. The repeatability analysis was performed as described above to calculate the RSD value of the peak area. The RSD of catechin was $1.83 \%(n=5)$, while that of quercetin was $1.57 \%(n=5)$.

\section{Stability test}

A test solution of a known concentration was prepared at room temperature and tested at 0,2 , 4,6 , and $8 \mathrm{~h}$ after preparation. Measured according to the chromatographic conditions, the RSD of the catechin content corresponded to $1.4 \%$, while that of quercetin was $1.2 \%$. The results showed that each test solution was stable within $8 \mathrm{~h}$ after preparation.

\section{Recovery test}

A total of $200 \mu \mathrm{L}$ of the samples were added to $50 \mu \mathrm{L}$ of a reference solution with a final concentration of $80 \mu \mathrm{g} / \mathrm{mL}$. The experiment was repeated five times. According to the chromatographic conditions, the recovery results were measured.

\section{High performance liquid chromatography- mass spectrometry (HPLC-MS) analysis}

An Agilent 1260 HPLC Diamonsi IC18 $(2.1 \mathrm{~mm} \times$ $150 \mathrm{~mm} \times 5 \mu \mathrm{m}$ ) was used in the analysis. The mobile phase consisted of methanol (solvent A) and pure water (solvent $B$ ), and the flow rate was set at $0.80 \mathrm{~mL} / \mathrm{min}$. The optimal gradient program started with $50 \%$ methanol at the time of injection and increased linearly to $70 \%$ methanol over $2 \mathrm{~min}$, which was maintained for 5 min. Subsequently, the gradient was increased linearly to $90 \%$ methanol over 2 min and held for $5 \mathrm{~min}$, followed by a decrease back to $50 \%$ acetonitrile over $0.1 \mathrm{~min}$ and held for another 5 min until the next injection. The temperature of the column oven was maintained at $30^{\circ} \mathrm{C}$, and the sample injection volume was $5 \mu \mathrm{L}$. The detection wavelengths used for catechin and quercetin were $\lambda_{1}=270 \mathrm{~nm}$ and $\lambda_{2}=368 \mathrm{~nm}$, respectively.

The MS analysis was performed using the Agilent 1100 series ion trap mass spectrometer with an electrospray ionization source in the positive ion detection mode. The following parameters were used: spray voltage, $4,000 \mathrm{~V}$; ionization temperature, $350{ }^{\circ} \mathrm{C}$; atomizer pressure, $35 \mathrm{psi}$; nitrogen flow rate, $8 \mathrm{~L} / \mathrm{min}$; and mass scan range, $200-800 \mathrm{~m} / \mathrm{z}$.

\section{Statistical analysis}

The data are expressed as mean \pm standard deviation (SD) and were analyzed using analysis of variance (ANOVA) and SPSS/PC (Statistical Package for Social Sciences). Statistical significance was set at $p<0.05$.

\section{RESULTS}

\section{Total FRTs}

The regression equation of rutin, which was used as the standard, was $y=10.639 x+0.0028(r=$ $0.9997)$. At concentrations of $0.01-0.07 \mathrm{mg} / \mathrm{mL}(\mathrm{r}$ $=0.9997$ ) for rutin, which showed a good linear relationship with the absorbance within the scope, the content of FRTs was $73.85 \%$; this was in agreement with the national standard. 


\section{Recovery}

The catechin and quercetin average recoveries were 98.08 and $98.50 \%$, respectively, while the RSD values were $1.98 \%$ for catechin and 1.36 $\%$ for quercetin. The results of the measurement recovery are summarized in Table 1.

\section{Qualitative analysis of catechin and quercetin}

According to the relevant literature and the mass to charge ratio of the compound, the retention time of catechin standard and FRT samples were comparable (Figure 1); this was also the case for quercetin. As shown in Figure 1, compound 1 contained a fragment of $\mathrm{m} / \mathrm{z} 291.0875$, and was consistent with the relative standard molecular weight of catechin (290); thus, compound 1 was catechin. As shown in Figure 1, compound 2 contained a fragment of $\mathrm{m} / \mathrm{z} 303.0425$, which is consistent with the relative standard molecular mass of quercetin (302.0); thus, compound 2 was quercetin.
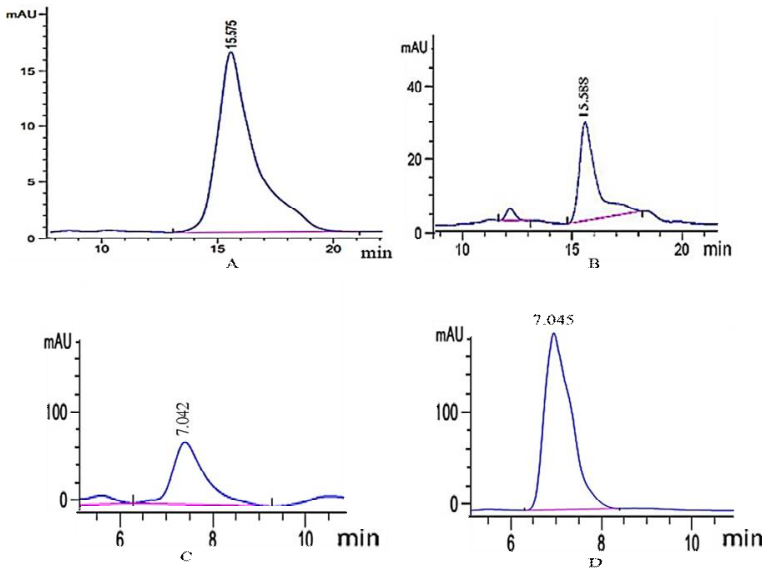

Figure 1: HPLC chromatograms of catechin and quercetin. (A) catechin standard; (B) flavonoid of Rosa roxburghii Tratt (FRT) samples; (C) quercetin standard; (D) FRT samples

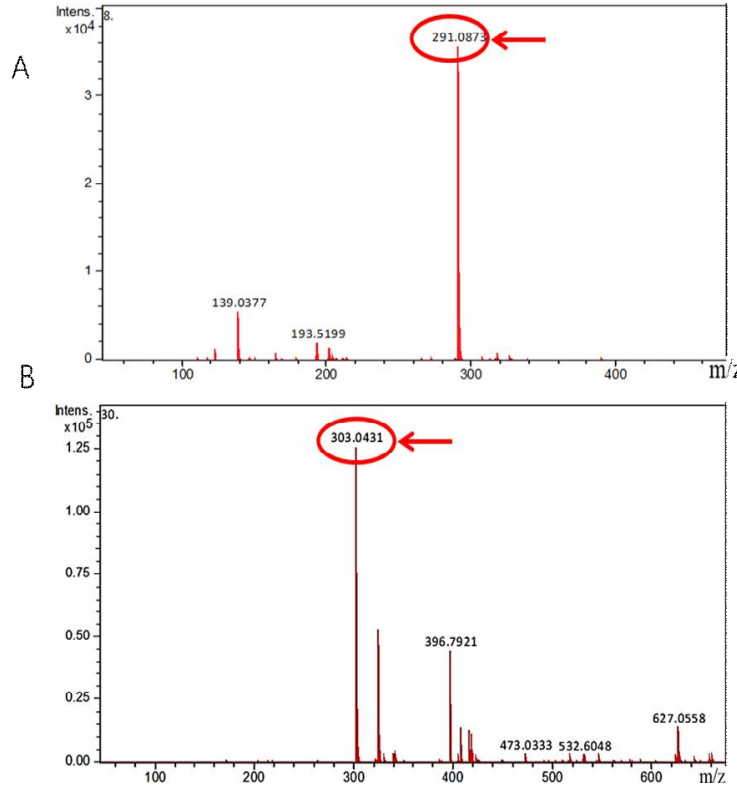

Figure 2: Mass spectra of compounds in flavonoid of Rosa roxburghii Tratt (FRT). (A) Mass spectrum of catechin in FRT; (B) mass spectrum of quercetin in FRT

\section{Contents of catechin and quercetin}

The test solution was measured to determine the catechin and quercetin peak areas under the same chromatographic conditions. The measurement was performed in triplicate. Comparison to the standard curve was used to calculate the content of catechin and quercetin, and the results are shown in Table 2 .

Table 2: Contents of catechin and quercetin $(n=3)$

\begin{tabular}{llll}
\hline Sample & $\begin{array}{l}\text { Content } \\
(\%)\end{array}$ & $\bar{X}$ & RSD (\%) \\
\hline Catechin & 37.26 & 34.26 & 2.88 \\
& 34.01 & & \\
Quercetin & 31.51 & & \\
& 2.65 & 2.97 & 1.49 \\
& 3.16 & & \\
& 3.10 & & \\
\hline
\end{tabular}

Table 1: Recovery data

\begin{tabular}{lcccccc}
\hline Compound & $\begin{array}{c}\text { Original } \\
\text { content } \\
(\boldsymbol{\mu} \mathbf{g} / \mathbf{m L})\end{array}$ & $\begin{array}{c}\text { Addition } \\
(\boldsymbol{\mu} \mathbf{g} / \mathbf{m L})\end{array}$ & $\begin{array}{c}\text { Measured } \\
\text { value } \\
(\boldsymbol{\mu} \mathbf{g} / \mathbf{m L})\end{array}$ & $\begin{array}{c}\text { Recovery } \\
(\%)\end{array}$ & $\begin{array}{c}\text { Average } \\
\text { recovery } \\
(\%)\end{array}$ & $\begin{array}{c}\text { RSD } \\
(\%)\end{array}$ \\
\hline Catechin & 110.45 & 20 & 130.06 & 98.05 & 98.08 & 1.98 \\
& 110.45 & 20 & 129.95 & 97.48 & & \\
Quercetin & 110.45 & 20 & 130.28 & 99.15 & & \\
& 112.76 & 20 & 131.81 & 95.25 & & \\
& 112.76 & 20 & 132.85 & 100.45 & & \\
& 20.45 & 20 & 40.12 & 98.35 & 98.05 & \\
& 20.45 & 20 & 40.38 & 99.65 & & \\
\hline
\end{tabular}

$\mathrm{RSD}=$ relative standard deviation 


\section{DISCUSSION}

Flavonoids are polyphenol compounds with 2phenylchromone as the mother nucleus. These compounds most often exist in a free state or in the form of sugar [21]. Flavonoids provide several beneficial properties, such as radioprotection, anti-free radical, antioxidant, antibacterial, antiviral, anticancer, and cancer prophylactic. In mice, flavonoids have been shown to increase significantly the 30-day survival of radiation and reduce apoptosis and necrosis induced by ionizing radiation $[22,23]$. Flavonoid protection mechanisms have many advantages, such as DNA protection, antioxidative effects, protection of the immune system, protection of the hematopoietic system, and mitigation of inflammation. The Rosa roxburghii Tratt bioactivity can be studied not only based on its flavonoids but also on its radioprotective activity of isolated monomeric compounds. It also has a good application prospect to compare the direct activity difference of different monomer components [24]. The ratio of methanol and water is very important when measuring isolated flavonoids. In this study, this approach was evaluated using various conditions including methanol-water $(10: 90)$, acetonitrilewater (10:90), methanol-0.1\% phosphoric acid buffer solution (10:90), and other mobile phases. Eventually, the gradient elution conditions started with $15 \%$ methanol at the time of injection, increased linearly up to $25 \%$ for $13 \mathrm{~min}$, and decreased subsequently back to $15 \%$ methanol, which was held for 10 min until the next injection. The advantage of this condition was that it could separate catechin and quercetin and had better peak shapes. Dual wavelength determination was used to measure catechin and quercetin at $368 \mathrm{~nm}$ and $270 \mathrm{~nm}$, respectively, which allowed maximum absorption, improved the resolution, and smoothed the baseline.

\section{CONCLUSION}

Accurate measurement of the levels of catechin and quercetin flavonoids in the fruit of Rosa roxburghii Tratt is important for further investigation of their benefits. The experimental determination of these two flavonoids may provide a theoretical basis and quality assessment for the rational development and utilization of Rosa roxburghii Tratt.

\section{DECLARATIONS}

\section{Acknowledgement}

This work was supported by the National Science Foundation of China (No. U1504824),
Department of Science and Technology Research Project of Henan Province in China (No. 142102310302), and Young Teachers Plan of Higher Schools in Henan Province (No. 2014GGJS-098).

\section{Conflict of interest}

The authors declare that no conflict of interest is associated with this work.

\section{Authors' contribution}

This work was performed by all authors named in this article, and all liabilities pertaining to claims relating to the content of this article will be borne by all of them.

\section{REFERENCES}

1. Wang $H$, Huang $C$, Liu SY, Du W. Determination of myricetin and quercetin in Rosa roxburghii Tratt by HPLC. Chi J of Exper Tradit Med Formulas 2013; 19: 109-111.

2. He $W P$, Zhu $X Y$. Research progress of bioactive components and product development of Rosa roxburghii Tratt. Guangxi Journal of Light Industry 2011; 11: 1-3.

3. An HM, Liu M, Yang M, Fan WG. Analysis of main organic acid composition in Rosa roxburghii Tratt. Science Agricultura Sinica 2011; 4: 2094-2100.

4. Tu GY, Liu LH. The nutrition components and health care medicinal in Rosa roxburghii Tratt. Forest By-Product and Specialty in China 2006; 1: 68-70.

5. Wang ZW, Hu XB, Wang K. Optimization of MicrowaveAssisted Extraction Process Parameters of Total Flavonoids from Rosa Roxburghii Tratt. Chinese Journal of Spectroscopy Laboratory 2012; 29: 1527-1530.

6. Zhang C, Liu X, Qing H, Li K, Wang J, Chen D, Zhuang $Y$. Inhibitory effects of rosa roxburghii Tratt juice on in vitro oxidative modification of low density lipoprotein and on the macrophage growth and cellular cholesteryl ester accumulation induced by oxidized low density lipoprotein. Clin Chim Acta 2001; 313: 37-43.

7. Zhang $X L, Q u W Q$, Sun $B, H u B$, Yang $X Y$. The Antioxidative Activity of Flavonids from Rosa roxburghii Tratt. Natural Product Research and Development 2005; 17: 396-400.

8. Tian HZ, Zhou YM, Zhao YB, Zang J, Hu R. Quantitative Analysis of Rutin in Rosa roxburghii Tratt Fruit by HPLC. Food sci 2009; 30: 203-205.

9. Dong LN, Pan SH. Research into the development of Rosa roxburghii Tratt. Jiangsu Journal of Traditional Chinese Medicine 2007; 39: 78-80.

10. Dai ZK, Yu LM, Yang XS, Shi JS. Three terpene compound $C L 1$ in vitro anti human endometrial adenocarcinoma in Rosa roxburghii Tratt. Lishizhen 
Medicine and Materia Medica Research 2011; 22: 16561659.

11. Dai ZK, Yu LM, Yang XS. Anticancer tudy of extract (CL) of Rosa Roxburghii Tratt. China Journal of Chinese Materia Medica 2007; 32: 1453-1456.

12. Dai ZK, Yang XS, Yu LM. The synergistic antitumor effect of 5-tluorouracil (5-FU) combined with extmet from Rosa Roxburghii Tratt on human hepatocellular carcinoma cell line SMMC-7721.Tumor2009; 29: 955-960.

13. Yu LW, Wang CZ, He RR. Protect effect of Rosa Roxburghii Tratt drink against liver injury in mice loaded with restraint stress. Journal of Food Science and Biotechnology 2010; 29: 730-734.

14. Wang ZW, Wei JH. Determination of rutin and quercetin in Rosa roxburghii Tratt by RP-HPLC. China Brewing 2014; 33: 109-111.

15. Yamane T, Nakatani H, Kikuoka N, Takahashi T, Iwata $Y$, Kitao $Y$, Oya K, Takahashi $T$. Inhibitory effects and toxicity of green tea polyphenols for gastrointestinal carcinogenesis. Cancer 1996; 77: 1662-1665.

16. Nakane H, Ono K. Differential inhibitory effect of some catechin derivatives on the activities of human immunodeficiency virus reverse transcriptase and cellular deoxyribonucleic and ribonueleic acid polymerases. Biochemistry 1990; 29: 2841-2843.

17. Wang GL, Yu JD, Tian JG, Zhang J. Study on chemical composition of catechu quantitative determination of catechin and epicatechin by RP-HPLC. Chinese Journal of Pharmaceutical Analysis 1999; 19: 88-92.

18. Zhang L, Duan HJ, Fang HJ, Wang ZS, Liu ZH. Analysis of catechins and alkaloids in HPLC in tea. Acta Pharmaceutica Sinica 1995; 30: 920-923.

19. Schurig V. Separation of enantiomers by gas chromatography. J Chromatogr A 2001; 906: 275-280.

20. Shi $X Y$, Liang $P$, Gao $X W$. The capillary gas chromatographic properties of four $\beta$-cyclodextrin derivatives with allyl groups or propyl groups on 3position or 6-position of $\beta$-cyclodextrin. Anal Chim Acta 2005; 548: 86-90.

21. $L i \quad Y N$, Zhang $W B$, Zhang $J H, X u P$, Hao $M H$. Radioprotective effect and other biological benefits associated with flavonoids [J]. Tropical Journal of Pharmaceutical Research 2016; 15(5):1099-1108.

22. Xu P, Jia JQ, Jia JF, Jiang EJ. Radioprotective effects of troxerutin against gamma irradiation in V79 Cells and Mice. Asian Pacific J Cancer Prev 2011; 12: 2593-2596.

23. Xu P, Zhang WB, Cai XH, Lu DD, He XY, Qiu PY, Wu J. Flavonoids of Rosa roxburghii Tratt act as radioprotectors. Asian Pac J Cancer Prev 2014; 15: 8171-8175.

24. Ha CT, Li XH, Fu D. Genistein nanoparticles protect mouse hematopoietic system and prevent proinflammatory factors after gamma irradiation. Radiat Res 2013; 180:316-325. 